\title{
Testing New Physics with Unitarity Triangle Fit
}

\author{
Marcella Bona* \\ INFN and Universitá di Torino, Italy \\ E-mail: bona@to.infn.it
}

\section{on behalf of UTfit Collaboration ${ }^{\dagger}$}

\begin{abstract}
We review here the status of the Unitarity Triangle beyond the Standard Model. Within the Standard Model (SM), all flavour and CP violating quark weak interactions are governed by the CKM matrix, which can be parameterized in terms of three angles and one phase, or, more conveniently, in terms of the parameters $\lambda, A, \bar{\rho}$ and $\bar{\eta}$. This implies very strong correlations among flavour and CP violating observables within the SM. The Unitarity Triangle (UT) is a very useful tool to analyze these correlations. With the recent data on $B$ decays, the UT fit has become strongly overconstrained, thus it is now possible to test the CKM mechanism within the SM and to derive constraints on New Physics (NP). In this talk, we will review the latter aspect, while the first is contained in ref. [1]. More details on the procedure and on the results can be found in ref. [2]. We analyze the determination of the unitarity triangle and the model-independent constraints on new physics that can be derived from this analysis. We find stringent bounds on new contributions to $K-\bar{K}$ and $B_{d}-\bar{B}_{d}$ mixing, pointing either to models of minimal flavour violation or to models with new sources of flavour and CP violation in $b \rightarrow s$ transitions. We also discuss the status of the universal unitarity triangle in minimal flavour violation. Finally, we perform a combined fit of the Unitarity Triangle and of new physics contributions in Minimal Flavour Violation, reaching a sensitivity to a new physics scale of about $5 \mathrm{TeV}$. More details on these analyses and on the results can be found in ref. [3].
\end{abstract}

International Europhysics Conference on High Energy Physics

July 21st - 27th 2005

Lisboa, Portugal

\footnotetext{
* Speaker.

${ }^{\dagger}$ UTfit Collaboration: M. Bona, M. Ciuchini, E. Franco, V. Lubicz, G. Martinelli, F. Parodi, M. Pierini, P. Roudeau, C. Schiavi, L. Silvestrini, A. Stocchi, and V. Vagnoni
} 


\section{Constraints on New Physics from the Unitarity Triangle}

Thanks to the measurements of the UT angles recently performed at $B$ factories, the UT fi $t$ is at present time overconstrained. Therefore, it has become possible to add NP contributions to all quantities entering the UT analysis and to perform a combined fi t of both NP and SM parameters. In general, NP models introduce a large number of new parameters whose specifi $\mathrm{c}$ list and the actual values can only be determined within a given model. Nevertheless, each of the meson-antimeson mixing processes is described by a single amplitude and can be parameterized, without loss of generality, in terms of two parameters, which quantify the difference between the full amplitude and the SM one. Thus, in the case of $B_{q}^{0}-\bar{B}_{q}^{0}$ mixing we defi ne $C_{B_{q}} e^{2 i \phi_{B_{q}}}=\left\langle B_{q}^{0}\left|H_{\text {eff }}^{\text {full }}\right| \bar{B}_{q}^{0}\right\rangle /\left\langle B_{q}^{0}\left|H_{\text {eff }}^{\mathrm{SM}}\right| \bar{B}_{q}^{0}\right\rangle$ with $q=d, s$, and where $H_{\mathrm{eff}}^{\mathrm{SM}}$ includes only the SM box diagrams, while $H_{\mathrm{eff}}^{\text {full }}$ includes also the NP contributions. In $K^{0}-\bar{K}^{0}$ mixing, we fi nd it convenient to introduce a single parameter relating the imaginary part of the amplitude to the SM one: $C_{\varepsilon_{K}}=\operatorname{Im}\left[\left\langle K^{0}\left|H_{\text {eff }}^{\text {full }}\right| \bar{K}^{0}\right\rangle\right] / \operatorname{Im}\left[\left\langle K^{0}\left|H_{\text {eff }}^{\mathrm{SM}}\right| \bar{K}^{0}\right\rangle\right]$. Therefore, all NP effects in $\Delta F=2$ transitions are parameterized in terms of three real quantities, $C_{B_{d}}, \phi_{B_{d}}$ and $C_{\varepsilon_{K}}$. NP in the $B_{s}$ sector is not considered, due to the lack of experimental information.
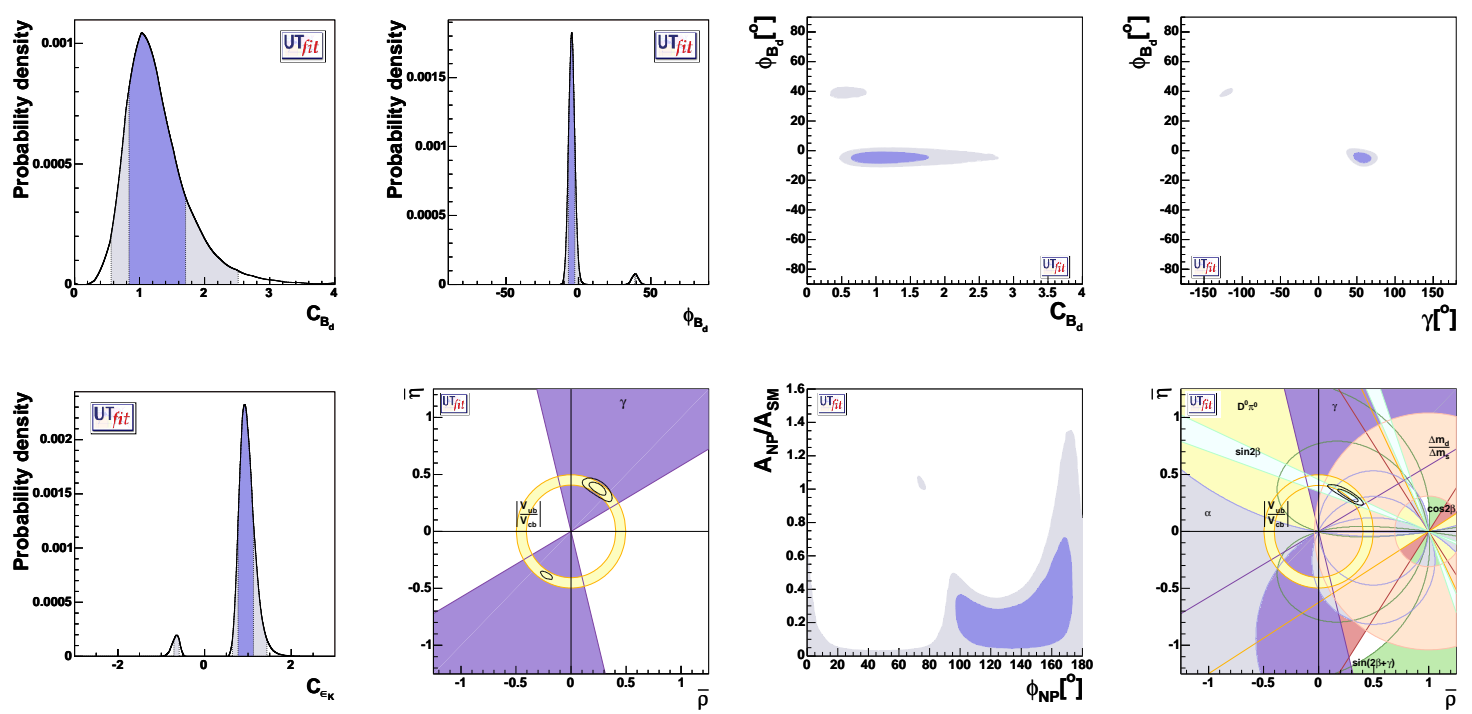

Figure 1: From top to bottom and from left to right, p.d.f.'s for $C_{B_{d}}, \phi_{B_{d}}, \phi_{B_{d}} v s . C_{B_{d}}, \phi_{B_{d}} v s . \gamma, C_{\varepsilon_{K}}$, the resulting selected region on the $\bar{\rho}-\bar{\eta}$ plane obtained from the NP analysis, P.d.f. in the $\left(A_{\mathrm{NP}} / A_{\mathrm{SM}}\right)$ vs. $\phi_{\mathrm{NP}}$ plane for NP in the $|\Delta B|=2$ sector, and the selected region on $\bar{\rho}-\bar{\eta}$ plane obtained from the determination of the UUT analysis [3].

We also include in the fi t NP effects in $\Delta B=1$ transitions that can also affect some of the measurements entering the UT analysis, in particular the measurements of $\alpha$ and $A_{\mathrm{SL}}$ [3]. The results obtained in a global $\mathrm{fi} \mathrm{t}$ for the NP parameters are shown in Fig. 1, together with the corresponding regions in the $\bar{\rho}-\bar{\eta}$ plane. Writing $C_{B_{d}} e^{2 i \phi_{B_{d}}}=\left(A_{\mathrm{SM}} e^{2 i \beta}+A_{\mathrm{NP}} e^{2 i\left(\beta+\phi_{\mathrm{NP}}\right)}\right) /\left(A_{\mathrm{SM}} e^{2 i \beta}\right)$ and given the p.d.f. for $C_{B_{d}}$ and $\phi_{B_{d}}$, we can derive the p.d.f. in the $\left(A_{\mathrm{NP}} / A_{\mathrm{SM}}\right)$ vs. $\phi_{\mathrm{NP}}$ plane as seen in Fig. 1. We see that the NP contribution can be substantial if its phase is close to the SM phase, while for arbitrary phases its magnitude has to be much smaller than the SM one. ${ }^{1}$ Assuming that the small

\footnotetext{
${ }^{1}$ Notice that, with the latest data, the $\mathrm{SM}\left(\phi_{B_{d}}=0\right)$ is disfavoured at $68 \%$ probability due to a slight disagreement
} 
but non-vanishing value for $\phi_{B_{d}}$ we obtained is just due to a statistical fluctuation, the result of our analysis points either towards models with no new source of fhvour and CP violation beyond the ones present in the SM (Minimal Flavour Violation, MFV [5, 6]), or towards models in which new sources of fhvour and CP violation are only present in $b \rightarrow s$ transitions. In the rest of this talk we will concentrate on the former possibility.

\section{Minimal Flavour Violation models}

We now specialize to the case of MFV. Making the basic assumption that the only source of flbvour and CP violation is in the Yukawa couplings [4], it can be shown that the phase of $|\Delta B|=2$ amplitudes is unaffected by NP, and so is the ratio $\Delta m_{s} / \Delta m_{d}$. This allows the determination of the Universal Unitarity Triangle independent on NP effects in the context of MFV models: it is based on $\left|V_{u b} / V_{c b}\right|, \gamma, A_{C P}\left(B \rightarrow J / \Psi K^{(*)}\right), \beta$ from $B \rightarrow D^{0} h^{0}, \alpha$, and $\Delta m_{s} / \Delta m_{d}[6]$. In the bottomright plot in Fig. 1 we show the allowed region in the $\bar{\rho}-\bar{\eta}$ plane for the UUT analysis. The corresponding values and ranges are reported in Tab. 1.

\begin{tabular}{ccccccc}
\hline & \multicolumn{2}{c}{ UUT analysis } & \multicolumn{4}{c}{ Minimal Flavour Violation analysis } \\
\hline & & & \multicolumn{2}{c}{ low/moderate $\tan \beta$} & \multicolumn{2}{c}{ large $\tan \beta$} \\
& $68 \%$ & $95 \%$ & $68 \%$ & $95 \%$ & $68 \%$ & $95 \%$ \\
\hline $\bar{\rho}$ & $0.259 \pm 0.068$ & {$[0.107,0.376]$} & $0.216 \pm 0.058$ & {$[0.109,0.361]$} & $0.231 \pm 0.067$ & {$[0.112,0.375]$} \\
$\bar{\eta}$ & $0.320 \pm 0.042$ & {$[0.241,0.399]$} & $0.351 \pm 0.032$ & {$[0.265,0.406]$} & $0.347 \pm 0.036$ & {$[0.254,0.404]$} \\
$\sin 2 \beta$ & $0.728 \pm 0.031$ & {$[0.668,0.778]$} & $0.733 \pm 0.027$ & {$[0.679,0.781]$} & $0.731 \pm 0.027$ & {$[0.673,0.781]$} \\
$\alpha\left[^{\circ}\right]$ & $105 \pm 11$ & {$[81,124]$} & $98.6 \pm 9.5$ & {$[81.6,121.7]$} & $101 \pm 11$ & {$[82,124]$} \\
$\gamma\left[^{\circ}\right]$ & $51 \pm 10$ & {$[33,75]$} & $57.6 \pm 9.1$ & {$[35.7,79.1]$} & $55 \pm 11$ & {$[34,74]$} \\
$(2 \beta+\gamma)\left[^{\circ}\right]$ & $98 \pm 12$ & {$[77,123]$} & $104 \pm 10$ & {$[80,122]$} & $102 \pm 12$ & {$[77,121]$} \\
$\Delta m_{s}\left[\mathrm{ps}^{-1}\right]$ & $20.6 \pm 5.6$ & {$[10.6,32.6]$} & $19.5 \pm 2.6$ & {$[15.0,31.7]$} & $22.6 \pm 5.4$ & {$[15.5,35.1]$} \\
\hline
\end{tabular}

Table 1: Results for UT parameters from both the UUT and the MFV generalized analyses [3].

If we now consider MFV models with one Higgs doublet or low/moderate $\tan \beta$, all NP effects in $|\Delta F|=2$ transitions are due to the effective Hamiltonian ${ }^{2}\left(a / 2 \Lambda^{2}\right)\left(\bar{Q}_{L} \lambda_{F C} \gamma_{\mu} Q_{L}\right)^{2}$ with $\left(\lambda_{F C}\right)_{i j}=$ $Y_{t}^{2} V_{t i}^{*} V_{t j}$ for $i \neq j$ and zero otherwise, $Y_{t}$ the top quark Yukawa coupling, $\Lambda$ the scale of NP and $a$ an unknown (but real) Wilson coeffi cient. The value of $a$ can range from order one for strongly interacting extensions of the SM to much smaller values for weakly interacting theories and/or symmetry suppressions analogous to the GIM mechanism in the SM. To project this onto the SM $|\Delta F|=2$ effective Hamiltonian, only a modifi cation of the top quark contribution to box diagrams has to be applied. Normalizing the NP Wilson coeffi cient to the SM effective electroweak scale $\Lambda_{0}=Y_{t} \sin ^{2} \theta_{W} M_{W} / \alpha \approx 2.4 \mathrm{TeV}$, we obtain $S_{0}\left(x_{t}\right) \rightarrow S_{0}\left(x_{t}\right)+\delta S_{0}$ and $\delta S_{0}=4 a\left(\Lambda_{0} / \Lambda\right)^{2}$. We can therefore determine simultaneously the shape of the UT and $\delta S_{0}$ from the standard UT analysis. Then, choosing as reference values $a= \pm 1$, we can translate the constraints on $\delta S_{0}$ into a lower bound on $\Lambda$. At $95 \%$ probability we obtain (see Fig. 2): $\Lambda>3.6(5.1) \mathrm{TeV}$ for $\delta S_{0}>0\left(\delta S_{0}<0\right)$.

between $\sin 2 \beta$ and $\left|V_{u b} / V_{c b}\right|$. This requires $A_{\mathrm{NP}} \neq 0$ and $\phi_{\mathrm{NP}} \neq 0$. For the same reason, $\phi_{\mathrm{NP}}>90^{\circ}$ at $68 \%$ probability and the plot is not symmetric around $\phi_{\mathrm{NP}}=90^{\circ}$.

${ }^{2}$ Here and in the rest of this section we follow the notation of Ref. [4]. 
Also in this case, we can obtain predictions for UT parameters, together with a constraint on NP contributions (see Tab. 1).

In the case of large $\tan \beta$, the situation changes since the bottom Yukawa coupling is not negligible anymore, and it can distinguish transitions involving $b$ quarks from those involving only light quarks. This spoils the correlation of $|\Delta B|=2$ with $|\Delta S|=2$ amplitudes, so that two uncorrelated parameters $\delta S_{0}^{B}$ and $\delta S_{0}^{K}$ are required in this case, to take into account NP contributions to $B_{d, s^{-}}$ $\bar{B}_{d, s}$ and $K-\bar{K}$ mixing. In a global fi t, made by using all the available inputs, $\Delta m_{d}$ and $\Delta m_{d} / \Delta m_{s}$ determine the value of $\delta S_{0}^{B}$, $\varepsilon_{K}$ fi xes $\delta S_{0}^{K}$, while $\bar{\rho}$ and $\bar{\eta}$ are given by the combination of all the other constraints.

Performing this analysis, we bound the UT parameters as given in Tab. 1 and we limit the NP scale to be, from $B_{d, s}-\bar{B}_{d, s}$ mixing $\Lambda>2.6(4.9) \mathrm{TeV}$ for $\delta S_{0}^{B}>0\left(\delta S_{0}^{B}<0\right)$ at $95 \%$ probability, and from $K-\bar{K}$ mixing $\Lambda>3.2(4.9) \mathrm{TeV}$ for $\delta S_{0}^{K}>0\left(\delta S_{0}^{K}<0\right)$ at $95 \%$ probability. The output distributions for $\delta S_{0}^{B}$ and $\delta S_{0}^{K}$ are given in Fig. 2.
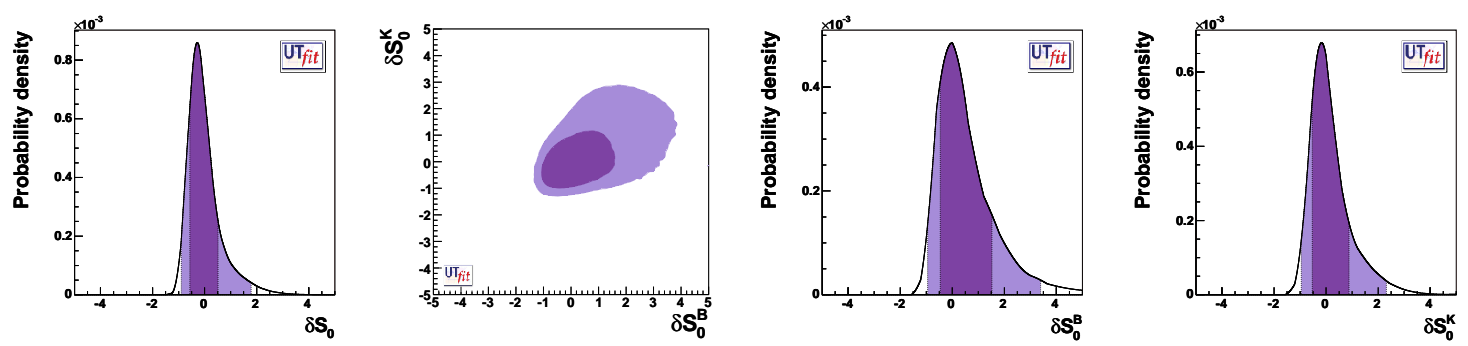

Figure 2: From left to right: P.d.f. of $\delta S_{0}, \delta S_{0}^{K}$ vs $\delta S_{0}^{B}, \delta S_{0}^{B}$ and $\delta S_{0}^{K}$. See the text for details.

It is instructive to observe the two-dimensional plot of $\delta S_{0}^{B}$ vs. $\delta S_{0}^{K}$ in Fig. 2: within models with only one Higgs doublet or with small $\tan \beta$, the two $\delta$ 's are bound to lie on the line $\delta S_{0}^{B}=\delta S_{0}^{K}$. The correlation coeffi cient $R$ provides a measure of this relation. We fi nd $R=0.52$ giving no compelling indication on the value of $\tan \beta$.

\section{References}

[1] M. Bona [on behalf of the UTfit Collaboration], talk given at this conference with the title "Status of the Unitarity Triangle in the Standard Model"

[2] M. Bona et al. [UTfit Collaboration], JHEP 0507 (2005) 028; www . ut fit . org.

[3] M. Bona et al. [UTfit Collaboration], arXiv:hep-ph/0509219, and references therein.

[4] G. D’Ambrosio et al., Nucl. Phys. B 645 (2002) 155.

[5] E. Gabrielli and G. F. Giudice, Nucl. Phys. B 433 (1995) 3 [Erratum-ibid. B 507 (1997) 549];

G. D’Ambrosio et al., Nucl. Phys. B 645 (2002) 155;

[6] A. J. Buras, P. Gambino, M. Gorbahn, S. Jager and L. Silvestrini, Phys. Lett. B 500 (2001) 161 [arXiv:hep-ph/0007085]. 